\title{
Progressive cavitating leukoencephalopathy
}

INSERM

\section{Source}

INSERM. (1999). Orphanet: an online rare disease and orphan drug data base.

Progressive cavitating leukoencephalopathy. ORPHA:139447

Progressive cavitating leukoencephalopathy is characterized by acute episodes of neurological deficit (ataxia, dysarthria, seizures) with irritability and opisthotonus followed by either steady deterioration or alternating periods of rapid progression and prolonged periods of stability. 